\title{
MODELO PREDICTIVO DE PREECLAMPSIA SEGÚN EL CONSUMO DE MACRONUTRIENTES MEDIANTE APRENDIZAJE AUTOMÁTICO EN UN HOSPITAL DE LIMA, 2019
}

\author{
Victor Hugo Moquillaza Alcántara ${ }^{1}$, Angélica María Guerrero Rosa²
}

\begin{abstract}
RESUMEN
Objetivo: Determinar un modelo predictivo de preeclampsia según el consumo de macronutrientes mediante aprendizaje automático en un hospital de Lima, 2019. Material y métodos: Estudio analítico de casos y controles donde participaron 120 gestantes atendidas en el Hospital Nacional Sergio Bernales. Se evaluó la preeclampsia de acuerdo con el registro clínico hospitalario y el consumo de macronutrientes en calorías ingeridas de lípidos, carbohidratos y proteínas. Se generaron puntos de corte predictivos de preeclampsia mediante un modelo de aprendizaje automático supervisado. Resultados: Las pacientes que presentaron preeclampsia presentaron menor consumo de carbohidratos $(\mathrm{Me}=1004.1 \mathrm{cal})$ y mayor consumo de lípidos $(\mathrm{Me}=1869.9 \mathrm{cal})$ y proteínas $(\mathrm{Me}=684.5 \mathrm{cal})$. De ellos solo existió variación significativa en carbohidratos y lípidos $(p<0.05)$. Mediante el método de decision tree se determinó que consumir menos o igual a 1743.57cal de lípidos o 1463.2cal por día reduce la probabilidad de presentar preeclampsia $(p<0.05)$. Conclusión: El consumo de lípidos y carbohidratos, medido en calorías, a ciertos valores permite predecir la presencia de preeclampsia mediante un modelo de aprendizaje automático.
\end{abstract}

Palabras clave: Preeclampsia, macronutrientes, modelo predictivo (Fuente: DeCS BIREME).

\section{PRE-CLAMPSIA PREDICTIVE MODEL ACCORDING TO THE USE OF MACRONUTRIENTS THROUGH AUTOMATIC LEARNING IN A LIMA HOSPITAL, 2019}

\begin{abstract}
Objective: To determine a predictive model of preeclampsia according to the consumption of macronutrients through machine learning in a hospital in Lima, 2019. Material and methods: Analytical study of cases and controls where 120 pregnant women attended at the Sergio Bernales National Hospital participated. Preeclampsia was evaluated according to the hospital clinical record and the consumption of macronutrients in calories ingested from lipids, carbohydrates and proteins. Predictive cut-off points of preeclampsia were generated using a supervised machine learning model. Results: Patients who had preeclampsia had lower carbohydrate consumption ( $\mathrm{Me}=1004.1 \mathrm{cal}$ ) and higher consumption of lipids $(\mathrm{Me}=1869.9 \mathrm{cal})$ and proteins $(\mathrm{Me}=684.5 \mathrm{cal})$. Of these there was only significant variation in carbohydrates and lipids $(p<0.05)$. Using the decision tree method, it was determined that consuming less than or equal to 1743.57cal of lipids or $1463.2 \mathrm{cal}$ per day reduces the probability of presenting preeclampsia $(p<0.05)$. Conclusion: The consumption of lipids and carbohydrates, measured in calories, at certain values allows to predict the presence of preeclampsia through a machine learning model.
\end{abstract}

Key word: Pre-eclampsia, macronutrients, predictive model (Source: MeSH NLM).

\section{INTRODUCCIÓN}

La preeclampsia es una patología hipertensiva del embarazo que representa la causalidad de una gran proporción de muertes maternas durante los últimos años en el Perú. Los estudios en la actualidad han abordado el tema desde un punto netamente clínico, aunque existen estudios que reportan que parte de su origen podría ser explicado desde el enfoque nutricional, tópico que los profesionales que atienden los controles prenatales no dominan en su totalidad. Por lo cual, en el presente estudio buscamos brindar indicadores predictivos generados mediante un proceso de Machine Learning.

La mortalidad materna es una problemática que trasciende la esfera médica debido a su implicancia social, económica, política y cultural; para lo cual el Perú ha desarrollado diversas políticas que han permitido su reducción, aunque aún persisten altas cifras ${ }^{1}$. De acuerdo con reportes del Ministerio de Salud peruano, la principal causa de muerte durante el 2018 fueron los trastornos hipertensivos. Asimismo, Lima Metropolitana ha sido el

\footnotetext{
Estudiante de Maestría Informática Biomédica, Universidad Peruana Cayetano Heredia, bachiller en Obstetricia de la Universidad Nacional Mayor de San Marcos

2 Bachiller en Obstetricia, Universidad Nacional Mayor de San Marcos. Lima, Perú
}

Citar como: Moquillaza Alcántara VH, Guerrero Rosa AM. Modelo predictivo de preeclampsia según el consumo de macronutrientes mediante aprendizaje automático en un hospital de lima, 2019. Rev Peru Investig Matern Perinat. 2019;8(4): 14-8 DOI https://doi.org/10.33421/inmp.2019168 
departamento con mayor frecuencia de muertes ${ }^{2}$. Por otro lado, la nutrición durante el embarazo llega a ser un tópico importante debido a que determina el desarrollo fetal y los problemas de salud crónicos en la madre y el recién nacido ${ }^{3}$. La evidencia reporta que gran parte de la falta de una inadecuada nutrición durante el embarazo se debe a los tabúes que se presentan ${ }^{4}$.

El profesional que participa en la atención prenatal carece de información respecto a los requerimiento nutricionales durante el embarazo ${ }^{5}$, por lo cual en el Perú se requiere la derivación a un servicio de nutrición. Sin embargo, esta atención especializada no se encuentra en muchos establecimientos rurales, lo cual podría explicar la alta prevalencia de gestantes con ganancia de peso excesiva ${ }^{6,7}$

A nivel nacional, el Instituto Nacional de Salud nos brinda una herramienta de conversión alimenticia que permite obtener el consumo de calorías diarias. Con lo cual, una herramienta que le permita al profesional de la salud conocer cuál es el consumo límite de ciertos macronutrientes que prevengan la preeclampsia permitiría una prevención primaria de esta patología hipertensiva. Para ello, en la actualidad se están utilizando métodos de inteligencia artificial (Machine Learning) a fin de prevenir múltiples patologías; sin embargo, su uso en preeclampsia aún es escaso ${ }^{8}$. Por lo cual, nos planteamos como objetivo determinar un modelo predictivo de preeclampsia según el consumo de macronutrientes mediante aprendizaje automático en un hospital de Lima, 2019.

\section{MATERIALES Y MÉTODOS}

\section{Diseño de estudio}

Estudio de enfoque cuantitativo, observacional, analítico de casos y controles.

\section{Población y muestra}

Se contó con la participación de 120 gestantes atendidas durante el periodo de abril a julio del 2019 en el Hospital Sergio Bernales, institución sanitaria del Ministerio de Salud ubicada en el distrito de Comas (Lima, Perú) con un nivel de complejidad III-1. Se incluyeron gestantes adultas ( $\geq 18$ años), con más de 20 semanas de gestación y que acepten voluntariamente participar. Se excluyeron a quienes presenten controles en otro establecimiento, tengan un embarazo múltiple y alguna discapacidad física o mental que impida su participación en el estudio. El cálculo del tamaño de muestra se realizó mediante el software OpenEpi considerando un nivel de confianza del $95 \%$, potencia del $80 \%$ y una razón de controles/casos de 2. Se utilizó un Odds Ratio de 3.3 y una proporción hipotética de controles expuestos del $57.14 \%{ }^{9}$. Con lo cual se obtuvo un tamaño de muestra de 120 gestantes, divididas en 40 casos (con preeclampsia) y 80 controles (sin preeclampsia). El muestreo fue de tipo probabilístico, aleatorio simple.

\section{Variables}

Se consideró como variable dependiente la presencia de preeclampsia, basada en el registro clínico; aunque fue verificada con los datos de hipertensión arterial y proteinuria. La variable independiente fue el consumo de macronutrientes, evaluado en base a las calorías (cal) por día consumidas en proteínas, carbohidratos y lípidos.
El consumo por día se obtuvo mediante un promedio aritmético de 2 días reportados y la conversión de alimentos consumidos a calorías se generó en base a las tablas de conversión del Instituto Nacional de Salud.

\section{Procedimientos}

Las participantes fueron enroladas al momento que ingresaron al servicio de hospitalización; en donde, de acuerdo con el diagnóstico del profesional de la salud, se clasificaron en quienes presentan preeclampsia o no. Luego de que la usuaria se encuentre estable y habiendo aceptado participar, se obtuvo la información respecto a la dieta de los últimos 2 días previos al ingreso al hospital, así como sus características sociodemográficas. Aquellas que ingresaron por inicio de trabajo de parto fueron abordadas en su etapa de puerperio, a fin de que no exista un sesgo de respuesta por la tensión de la situación que atraviesa.

\section{Consideraciones éticas}

El estudio contó con la revisión y aprobación del comité de investigación del Hospital Nacional Sergio Bernales (Oficio 790-2019-DG-HNSEB). Asimismo, se hizo uso del consentimiento informado para garantizar la participación voluntaria e informada. Todo dato obtenido fue codificado y nunca se solicitó información identificable, a fin de mantener la confidencialidad.

\section{Análisis estadístico}

Inicialmente se evaluó la calidad de los datos (evitar missing data o errores de tipeo) en una base de Excel, la cual luego fue exportada a un software estadístico. En STATA se realizó el análisis descriptivo, en donde las variables categóricas fueron reportadas mediante porcentajes y las variables numéricas mediante medidas de tendencia central y dispersión. Para evaluar asociaciones se utilizaron pruebas de Chi Cuadrado, T de Student y U de Mann-Whitney. Para todo análisis se consideró un nivel de confianza del $95 \%$.

En el software $\mathrm{R}$ se realizó un análisis de aprendizaje automático supervisado denominado "decision tree" (árbol de decisión). El análisis mediante decision tree nos permite generar puntos de corte en variables numéricas (V. Independiente) para determinar la probabilidad de presencia de una variable categórica (V. Dependiente). Se consideró un nivel de confianza del 95\%.

\section{RESULTADOS}

Las gestantes participantes presentaron una edad promedio de $26.87 \pm 6.49$ años, donde el $33.33 \%$ tuvo entre 21 a 25 años. El distrito de procedencia más prevalente fue Comas $(62.5 \%)$ y el estado civil más frecuente el de conviviente $(81.67 \%)$. Por otro lado, el $75 \%$ presentó secundaria completa y en su mayoría fueron católicos. Existió $66.67 \%$ que refirió obtener un ingreso económico mensual entre 500 a 1000 nuevos soles. Solamente el ingreso económico mostró una asociación con la presencia de preeclampsia $(p=0.026)$. Al evaluar el consumo de macronutrientes en kilocalorías se halló que la media variaba entre 3000 a 3750 calorías (cal). Asimismo, en la Figura 1 se observa que el consumo va disminuyendo a medida que progresa el embarazo. 


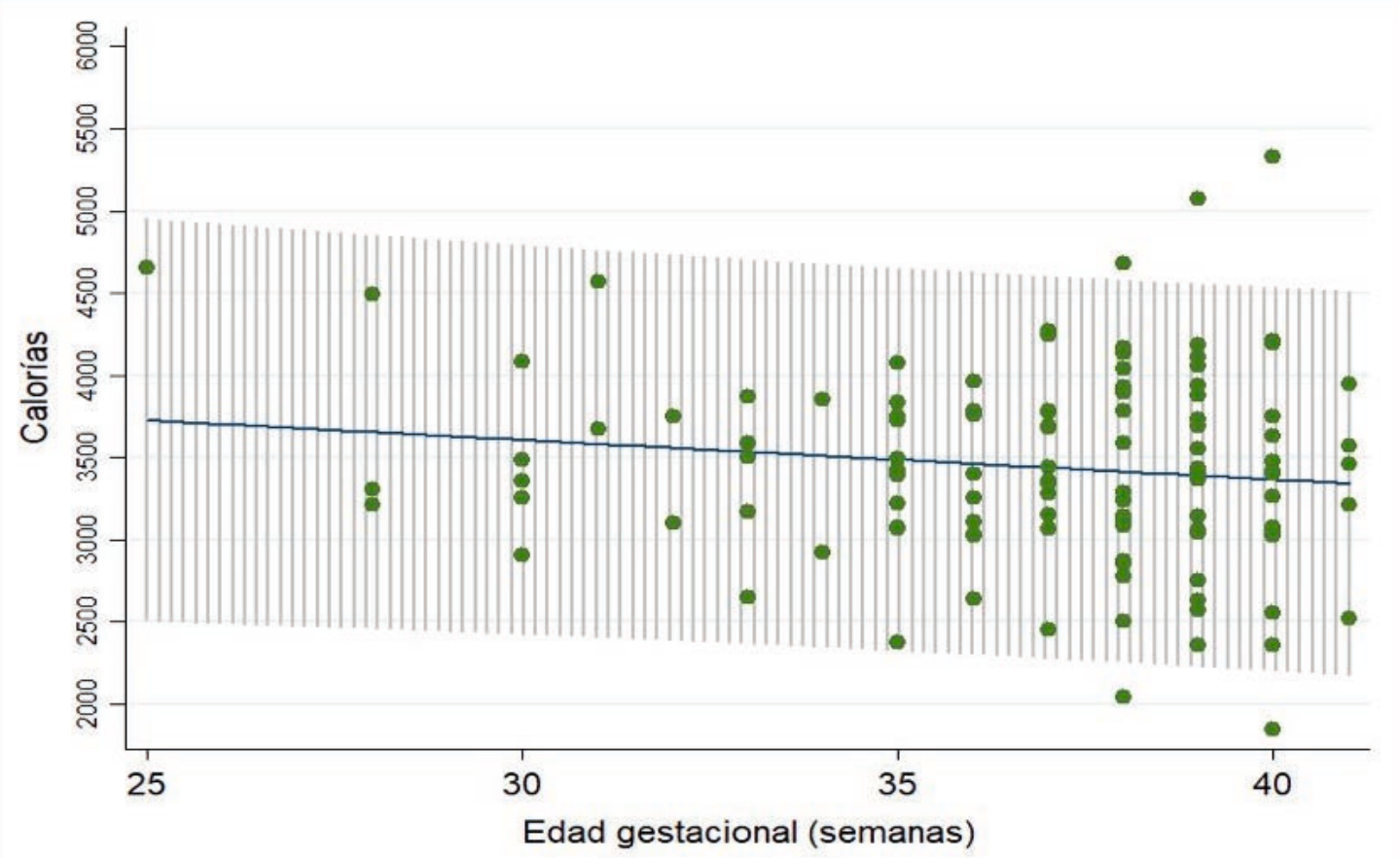

Figura 1. Consumo de macronutrientes (en kilocalorías) de acuerdo con la edad gestacional de gestantes atendidas en el Hospital Sergio Bernales, 2019

Asimismo, en la Tabla 2 se evaluó el consumo de calorías de los 3 macronutrientes ante la presencia de preeclampsia. En donde se observó que la mediana de consumo de carbohidratos fue de 1046.9 cal, en lípidos fue $1109.4 \mathrm{cal}$ y en proteínas fue $659.1 \mathrm{cal}$. Asimismo, quienes presentaban preeclampsia consumían significativamente menos carbohidratos $(p=0.017)$ y más lípidos $(p<0.001)$. El consumo de proteínas no se vio asociado a la presencia de preeclampsia.

Por último, se buscó determinar un número exacto de calorías, por macronutriente asociado a preeclampsia (carbohidratos y lípidos), a consumir por parte de la gestante para reducir la probabilidad de presentar preeclampsia. El modelo se realizó con 102 datos de entrenamiento y 18 de validación. En la Figura 2 se observa el análisis referente a los lípidos, donde se obtuvo que consumir menos o igual a $1743.57 \mathrm{cal}$ de lípidos por día reduce la probabilidad de presentar preeclampsia hasta un $17.81 \%$, mientras que consumir más de la cantidad establecida incrementa la probabilidad de presentar preeclampsia hasta un $68.97 \%$. Por otro lado, la Figura 3 muestra el análisis referente a carbohidratos; donde el consumir más de 1463.2cal de carbohidratos por día reduce la probabilidad de presentar preeclampsia hasta un $11.11 \%$, mientras que consumir menos de dicha cantidad incrementa la probabilidad de presentar preeclampsia hasta un $43.94 \%$.

Tabla 2. Consumo diario de macronutrientes asociado a preeclampsia en gestantes atendidas en el Hospital Sergio Bernales, 2019

\begin{tabular}{|c|c|c|c|c|c|c|c|}
\hline & \multirow{2}{*}{\multicolumn{2}{|c|}{ General }} & \multicolumn{4}{|c|}{ Preeclampsia } & \multirow{3}{*}{$\mathrm{p}$ valor ${ }^{t}$} \\
\hline & & & \multicolumn{2}{|c|}{ Presenta } & \multicolumn{2}{|c|}{ No presenta } & \\
\hline & $\mathrm{Me}$ & RIQ & $\mathrm{Me}$ & RIQ & $\mathrm{Me}$ & RIQ & \\
\hline \multicolumn{8}{|l|}{ Consumo (Cal) } \\
\hline Carbohidratos & 1046.9 & 1015.3 & 1004.1 & 234.9 & 1434.1 & 1054.1 & 0.017 \\
\hline Lípidos & 1109.4 & 994.2 & 1869.9 & 1227.2 & 1007.1 & 478.2 & $<0.001$ \\
\hline Proteínas & 659.1 & 437.4 & 684.5 & 278.1 & 645.9 & 540.8 & 0.515 \\
\hline
\end{tabular}

Consumo diario promedio basado en los 2 días previos al diagnóstico

† Evaluado mediante la prueba $U$ de Mann-Wh 


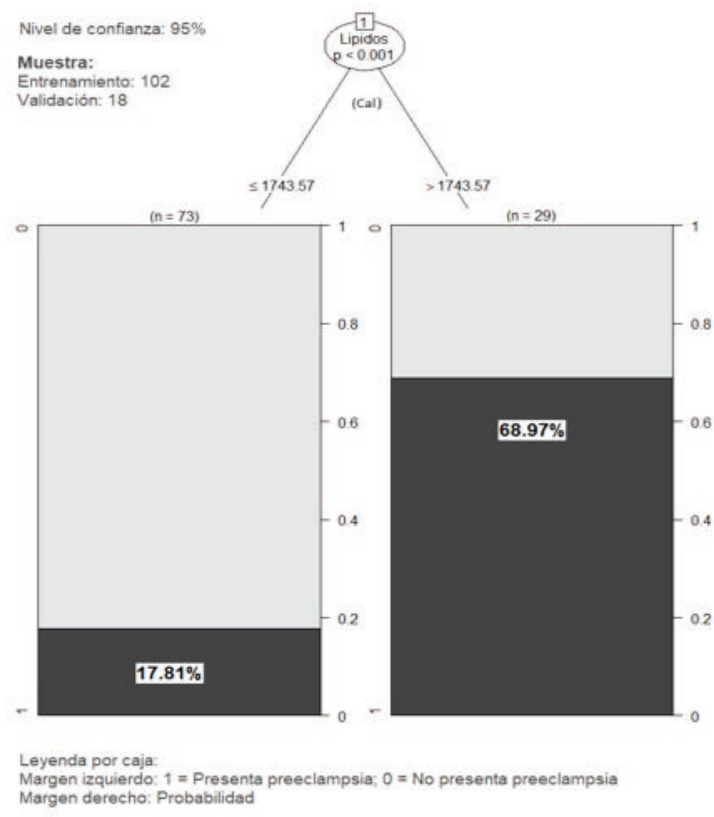

Figura 2. Punto de corte del consumo diario (en calorías) de lípidos para desarrollar preeclampsia

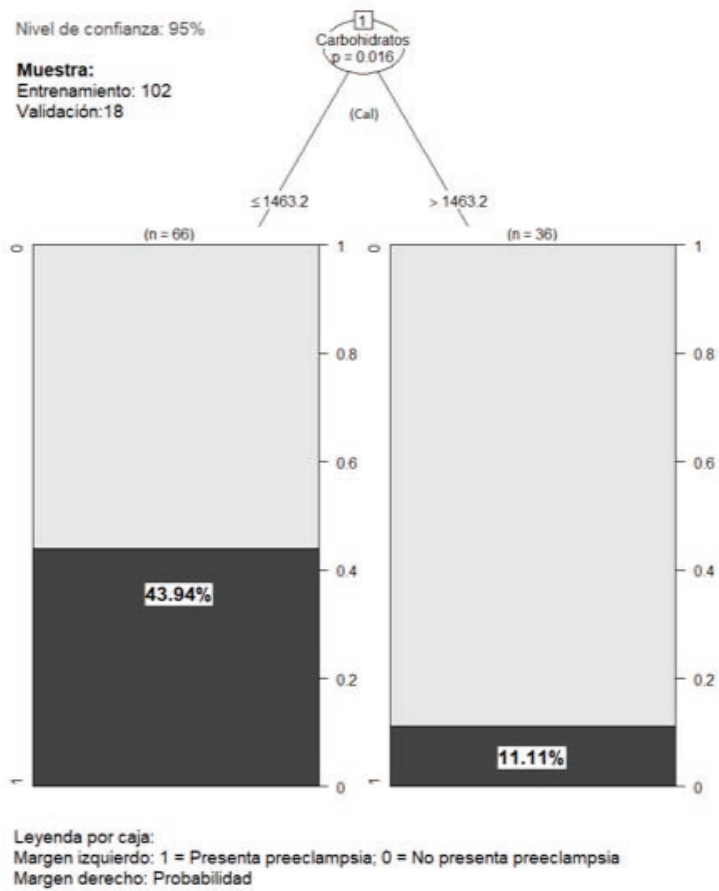

Figura 3. Punto de corte del consumo diario (en calorías) de carbohidratos para desarrollar preeclampsia

\section{DISCUSIÓN}

En el presente estudio se ha determinado que el consumo de lípidos se asocia significativamente con la preeclampsia, ya que las gestantes que presentaron esta patología tuvieron un consumo previo mayor que las gestantes sanas. Sin embargo, un estudio previo que comparó las concentraciones de lípidos en gestantes con y sin preeclampsia, tuvo como resultado que estas fueron más bajas en los casos de preeclampsia ${ }^{10}$. Al respecto, existen revisiones que cuestionan esta premisa, indicando que la asociación entre lípidos (específicamente el omega-3) y preeclampsia ha presentado hasta el momento limitaciones metodológicas, con lo cual se requieren mayores evidencias para resolver este vacío en el conocimiento ${ }^{11}$

Por otra parte, el consumo de carbohidratos también se asoció significativamente; en donde las que tuvieron preeclampsia mostraron un consumo previo menor de quienes no la tuvieron. Esto puede deberse a que en el período de gestación se requieren mayores macronutrientes, pero son los carbohidratos los que aportan mayor cantidad de energía ${ }^{12}$. Sin embargo, no se han hallado estudios previos que puedan explicar la asociación de ellos con preeclampsia, por lo cual se requieren mayores estudios que involucren a los carbohidratos dentro del análisis.

Finalmente, en el análisis que se realizó se definieron puntos de corte del consumo de lípidos y carbohidratos que permiten predecir la presencia de preeclampsia. Aunque el consumo de lípidos es lo que determina finalmente la presencia de preeclampsia. Este resultado concuerda con evidencia previa donde se menciona que durante la gestación ocurre una elevación progresiva de lípidos en la sangre, además indica que este macronutriente tiene un papel en el daño celular endotelial, que es un síntoma característico de la preeclampsia ${ }^{13}$. Sin embargo, un estudio realizado en los Estados Unidos examinó la relación entre los niveles de lípidos y la preeclampsia mediante predisposición genética, donde no se encontró una asociación estadísticamente significativa ${ }^{14}$.

Finalmente, concluimos que el consumo de lípidos y carbohidratos, medido en calorías, a ciertos valores permite predecir la presencia de preeclampsia mediante un modelo de aprendizaje automático.

\section{CONTRIBUCIONES A LA SALUD MATERNA PERINATAL}

El presente estudio permite conocer puntos de corte predictivos de preeclampsia en base a la alimentación diaria de la gestante, con lo cual se podrían utilizar este conocimiento en los controles prenatales y de este modo hacer derivaciones oportunas, antes de que se presente el trastorno hipertensivo.

Financiamiento: Concurso de Investigacion INMP 2019

Conflictos de interés: Los autores declaran no presentar conflicto de interés.

\section{REFERENCIAS BIBLIOGRÁFICAS}

1. Avila-Jaquez C, Avila-Jaquez C. Disminución de la mortalidad materna en Perú y el enfoque de capacidades. Convergencia [Internet]. 2019; 26(80):1-24 DOI: http://dx.doi.org/10.29101/ crcs.v26i80.10790

2. Ministerio de Salud. Boletín epidemiológico del Perú. Dirección General de Epidemiología. 2018; 27(1):1285-1290 Disponible en: https://www.dge.gob.pe/portal/docs/vigilancia/ boletines/2018/44.pdf

3. Procter SB, Campbell CG. Position of the Academy of Nutrition and Dietetics: nutrition and lifestyle for a healthy pregnancy outcome. J Acad Nutr Diet. julio de 2014;114(7):1099-103. DOI: http://dx.doi.org/10.1016/j.jand.2014.05.005 
4. Vasilevski V, Carolan-Olah M. Food taboos and nutritionrelated pregnancy concerns among Ethiopian women. J Clin Nurs. octubre de 2016;25(19-20):3069-75. DOI: http://dx.doi. org/10.1111/jocn.13319

5. Arrish J, Yeatman H, Williamson M. Midwives and nutrition education during pregnancy: a literature review. Women Birth J Aust Coll Midwives. 2014; 27(1):2-8. DOI: http://dx.doi. org/10.1016/j.wombi.2013.02.003

6. Cervantes Ramírez DL, Haro Acosta ME, Ayala Figueroa RI, Haro Estrada I, Fausto Pérez JA. Prevalencia de obesidad y ganancia de peso en mujeres embarazadas. Aten Fam. 2019;26(2):43-7. DOI: http://dx.doi.org/10.22201/ facmed.14058871p.2019.2.68824

7. Soria-Gonzales L. Ganancia de peso durante el embarazo y peso del recién nacido en puérperas atendidas en el Hospital San Juan de Lurigancho junio-julio 2018. Universidad San Martín Porres [Internet]. 2019. Disponible en: http://www. repositorioacademico.usmp.edu.pe/handle/usmp/4393

8. Jhee JH, Lee S, Park Y, Lee SE, Kim YA, Kang S-W, et al. Prediction model development of late-onset preeclampsia using machine learning-based methods. Plos One. 2019;14(8):e0221202. DOI: https://doi.org/10.1371/journal. pone.0221202

9. Ñaupari_ss.pdf [Internet]. [citado 23 de septiembre de 2019]. Disponible en: http://cybertesis.unmsm.edu.pe/ bitstream/handle/cybertesis/4704/\%C3\%91aupari_ ss.pdf?sequence=1\&isAllowed=y

10. Naupari S. Influencia de los hábitos alimentarios sobre la preeclampsia en gestantes que asisten al Instituto Nacional Materno Perinatal. Tesis, Universidad Nacional Mayor de san Marcos. Lima, Perú. 2019. Disponible en: https://www. redalyc.org/pdf/473/47333055005.pdf
11. Burchakov DI, Kuznetsova IV, Uspenskaya YB. Omega-3 Long-Chain Polyunsaturated Fatty Acids and Preeclampsia: Trials Say "No," but Is It the Final Word? Nutrients [Internet]. 15 de diciembre de 2017 [citado 3 de octubre de 2019];9(12). Disponible en: https://doi.org/10.3390/nu9121364

12. Orane A. Requerimientos nutricionales en el embarazo. Rev Clin de la Escuela de Medicina. 2016: 6(6). Disponible en: https://www.medigraphic.com/pdfs/revcliescmed/ucr-2016/ ucr164h.pdf

13. Duarte-Mote J, Perez-Torres C, Espinosa-López R, EngCastro VL, Calvo-Colindres J, Sánchez-Rojas G. Relación de dislipidemias y gravedad en preeclampsia. Rev Med Inst Mex Seguro Soc. 2014; 52(5): 516-20. Disponible en: https://www. medigraphic.com/pdfs/imss/im-2014/im145I.pdf

14. Spracklen CN, Saftlas AF, Triche EW, Bjonnes A, Keating B, Saxena R, et al. Genetic Predisposition to Dyslipidemia and Risk of Preeclampsia. Am J Hypertens. julio de 2015;28(7):915-23

\section{Correspondencia:}

Victor Hugo Moquillaza Alcántara

Direccción: Av. Honorio Delgado 430, San Martín de Porres. Lima, Perú.

Correo: victor.moquillaza@upch.pe

Teléfono: 982065404 